\title{
A numerical taxonomic study of the family Zygophyllaceae from Egypt
}

Kadry N. Abdel khalik ${ }^{1,2}$

Recebido em 4/10/2011. Aceito em 25/01/2012

\begin{abstract}
RESUMO
(Um estudo de taxonomia numérica da família Zygophyllaceae do Egito). Um estudo sistemático de 29 táxons pertencentes a sete gêneros das subfamílias Balanitoideae, Zygophylloideae, Peganoideae, Tribuloideae, Seetzenioideae eTetradiclidoideae das Zygophyllaceae do Egito foi realizado por meio de análise numérica, baseada em 61 caracteres morfológicos, incluindo partes vegetativas, grãos de pólen e sementes. Com base no agrupamento UPGMA e análise PCO, seis grupos principais são reconhecidos. Representantes destes grupos são agrupados com base em características com cargas fatoriais elevadas na análise PCO. Os resultados indicam que as Zygophyllaceae são um grupo heterogêneo, incluindo Peganum harmala que foi proposto para fazer parte de uma família separada. Zygophylloideae, Tribuloideae, Tetradiclidoideae e Seetzenioideae são os grupos mais homogêneos. Seções e grupos em ambos os gêneros Tribulus e Fagonia parecem ser artificiais.
\end{abstract}

Palavras-chave: Zygophyllaceae, Balanitoideae, Zygophylloideae, Peganoideae, Tribuloideae, Seetzenioideae, Tetradiclidoideae, Taxonomia numérica, UPGMA, PCO

\begin{abstract}
(A numerical taxonomic study of the family Zygophyllaceae from Egypt). A systematic study of 29 taxa belonging to 7 genera of subfamilies Balanitoideae, Zygophylloideae, Peganoideae, Tribuloideae, Seetzenioideae and Tetradiclidoideae of Zygophyllaceae from Egypt was carried out by means of numerical analysis based on sixty-one morphological characters, including vegetative parts, pollen grains and seeds. On the basis of UPGMA clustering and PCO analysis, six main groups are recognized. Representatives of these groups are clustered together based on characters with high factor loadings in the PCO analysis. The results indicate that Zygophyllaceae are heterogeneous, including Peganum harmala which has been proposed to belong in a separate family. Zygophylloideae, Tribuloideae, Tetradiclidoideae and Seetzenioideae are the most homogeneous groups. Sections and groups in both Tribulus and Fagonia seem artificial.
\end{abstract}

Key words: Zygophyllaceae, Balanitoideae, Zygophylloideae, Peganoideae, Tribuloideae, Seetzenioideae, Tetradiclidoideae, Numerical taxonomy, UPGMA cluster, PCO

\section{Introduction}

Zygophyllaceae is a widespread family of some 27 genera and 285 species subdivided into five subfamilies (Sheahan \& Chase 1996; 2000). It consists of herbs, shrubs and trees growing in arid and semi-arid areas in the tropics and subtropics. Earlier studies place the Zygophyllaceae in different orders, e.g. Sapindales, Rutales, Polygalales, Linales, and Geraniales (Cronquist 1968; Takhtajan 1969; 1980; 1983; 1986; Thorne 1992). Soltis et al. (2000) put the Zygophyllaceae and Krameriaceae together in their own order Zygophyllales within the eurosid I group, and this position is changed in APG III (2009). They put it within Fabids group. Delimitation of taxa within the family has repeatedly changed over time, because of their diversity in structural detail, particularly in Balanites, Nitraria, Peganum and Tetradiclis. For example, Engler (1896a; 1931) divided the family into seven subfamilies, 8 tribes and 4 subtribes: Peganoideae, Tetradiclidoideae, Chitonioideae, Augeoideae, Zygophylloideae, Nitrarioideae and Balanitoideae. He considered that Zygophylloideae (including the tribuloid genera) formed the central typical group, with Augeoideae based on the characters of the leaves and fruits.

Pollen morphology of the family has been also examined by Erdtman (1952), Shimakura (1973), Kuprianova \& Alyoshina (1978), Yunus \& Nair (1988) and Perveen \& Qaiser (2006). Sheahan \& Cutler (1993) investigated the vegetative anatomy of 37 species in 19 genera, and

\footnotetext{
${ }^{1}$ Umm Al-Qura University, Faculty of Science, Biology Department, Mecca, Saudi Arabia

${ }^{2}$ Sohag University, Faculty of Science, Botany Department, Sohag, Egypt

Author for correspondence: kadry3000@yahoo.com
} 
illustrated that there is anatomical evidence to exclude of Balanites into a separate family and they recommended that the tribuloid genera Tribulus, Kallstroemia and Kelleronia should be separated from the Zygophylloid genera at least at subfamily level. Sheahan \& Chase (1996) examined the phylogenetic analysis of Zygophyllaceae based on morphology, anatomy and $r b c \mathrm{~L}$ DNA sequence. Zygophyllaceae form a clade. They divided Zygophyllaceae into five subfamilies, whereas Nitraria and Peganum were separated from Zygophyllaceae. Eventually, Sheahan \& Chase (2000) analysed both $r b c \mathrm{~L}$ and $\operatorname{trn} \mathrm{L}-\mathrm{F}$ sequences from 36 members of Zygophyllaceae. The results confirmed the previous division of Zygophyllaceae into five subfamilies, and Zygophylloideae were strongly supported as monophyletic, whereas Zygophyllum turned to be a polyphyletic genus.

Beier et al. (2003), using non-coding trnL plastid data for 43 species of Zygophylloideae investigated the phylogeney of the family, retrieving a monophyletic subfamily Zygophylloideae, and the genus Zygophyllum, together with the genera Augea, Tetraena and Fagonia, turned to be a paraphyletic. In the flora of Egypt Zygophyllaceae is represented by six genera belonging to three subfamilies: Peganoideae Engl., Tetradiclidoideae Engl., and Zygophylloideae (Tackholm 1974; El Hadidi 1972), but recently Boulos (2000) recorded five genera. Systematics of Zygophyllaceae may be difficult because only leave or fruit morphological characters are traditionally in use by taxonomists. These characters are variable even within genera, or differ with one another in their distribution patterns among genera and may not reflect natural groups.

The aim of present study was to confirm the phenetic relationships between genera within the family in Egypt based on a large number of characters (61) with the use of numerical taxonomy and to check whether these results correspond to the systematics of the genera proposed by Engler (1896a; 1931), El Hadidi (1975) and Sheahan \& Chase $(1996 ; 2000)$

\section{Materials and methods}

\section{Plant material}

The present study is largely based on herbarium material kept in the following herbaria: CAI, CAIM, and SHG. A fresh material of most of the taxa from several localities in Egypt and Saudi Arabia was also studied. In the analyses, species constituted the OTU (Operational Taxonomic Unit) see Appendix 1. In order to broadly sample the variation, the OTU's consist of a number of collections (either herbarium specimens or fresh material or both) from different localities in Egypt. For some species from Egypt few specimens were available, in that case specimens from Saudi Arabia were used (Table 1).

\section{Morphological characters observations}

Table 2 shows the characters and character states scored for plant, pollen, and seed morphology, averaged for each OTU. In total 61 characters were taken into consideration, comprising 13 quantitative and 48 qualitative characters. Twelve of the qualitative characters were scored as binary and the remaining were scored as multi-state characters. Eleven characters from the 61 characters were treated as invariable characters and the rest are variable characters.

\section{Vegetative parts, flower and fruit characters}

The measurements for all specimens of a taxon were averaged into one OTU score for each of the characters. OTU scores for quantitative characters were arithmetic means based on at least 3 specimens (whenever possible). Because herbarium specimens cannot be considered to be a random sample of the species, we followed Wieringa (1999: 62-65) by calculating the mean of the minimum and maximum measurement. For some of the OTU's observations for some of the characters were not available, and these omissions were coded as missing data (-999). The complete data matrix is available on request at the Botany Department, Faculty of Science, Sohag University, Egypt.

\section{Pollen grain and seed characters}

The data of pollen morphology were mainly obtained from Erdtman (1952); Agababyan (1964); El Hadidi (1966); Yunus \& Nair (1988); and Perveen \& Qaiser (2006). Dried mature seeds were first examined by light microscope (Olympus type $\mathrm{BH}-2$ ), and 5-10 seeds for each taxon were selected to cover the range of variation when available. Seeds were mounted on stubs with double adhesive tape. The stubs were sputter-coated with gold/palladium for $3 \mathrm{~min}$. in a EMITECH K550. After coating, the specimens were examined with a Jeol-6300 scanning electron microscope, using accelerating voltages at $15-20 \mathrm{KV}$.

\section{Data analysis}

Two types of analyses were performed with NTSYS-pc 2.02k software (Applied Biostatistics Inc., Setauket, New York, USA). Firstly, I performed a cluster analysis using average taxonomic distance and UPGMA clustering (procedures SIMINT, SAHN, and TREE). To reduce the effects of different scales of measurement for different characters, the values for each character were standardized with procedure STAND, according to the formula: yI,STD = (yi- AVGyi)/ STDyi), Where the default value in NTSYS-pc (STAND) for $\mathrm{yi}=$ the value to be standardized, $\mathrm{AVGyi}=$ the average of all values for the character, and STDyi $=$ the standard deviation. The cophenetic correlation coefficient between the distance matrix and the tree matrix was calculated to examine the goodness of fit of the cluster analysis to the distance matrix (procedures $\mathrm{COPH}$ and MXCOMP). Secondly, a principal coordinates analysis (PCO) was performed, using the product-moment correlation as a coefficient. The procedure SIMINT was used to calculate the distance matrix based on 
Table 1. List of taxa used in the study (Boulos 2000), a comparison of most traditional (Engler 1896a, 1931; El Hadidi 1975) and phylogenetic classifications (Sheahan \& Chase 1996, 2000) are shown.

\begin{tabular}{|c|c|c|c|c|c|}
\hline $\mathrm{N}^{\circ}$ & Taxon & Engler $(1896 a, 1931)$ & El Hadidi (1975) & $\begin{array}{l}\text { Sheahan \& Chase } \\
\quad(1996,2000)\end{array}$ & $\begin{array}{c}\mathrm{N}^{\mathrm{o}} \text { of } \\
\text { individuals }\end{array}$ \\
\hline 1 & Balanites aegyptiacus Delile & Subfam. Balanitoideae & Family Balanitaceae & Subfam. Tribuloideae & 8 \\
\hline 2 & Fagonia arabica $\mathrm{L}$. & Subfam. Zygophylloideae & Subfam. Zygophylloideae & Subfam. Zygophylloideae & 10 \\
\hline 3 & F. boveana (Hadidi) Hadidi \& El-Garf & Subfam. Zygophylloideae & Subfam. Zygophylloideae & Subfam. Zygophylloideae & 9 \\
\hline 4 & F. bruguieri DC. Burm. Boiss & Subfam. Zygophylloideae & Subfam. Zygophylloideae & Subfam. Zygophylloideae & 10 \\
\hline 5 & F. cretica $\mathrm{L}$. & Subfam. Zygophylloideae & Subfam. Zygophylloideae & Subfam. Zygophylloideae & 8 \\
\hline 6 & F. glutinosa Delile & Subfam. Zygophylloideae & Subfam. Zygophylloideae & Subfam. Zygophylloideae & 8 \\
\hline 7 & F. indica Burm & Subfam. Zygophylloideae & Subfam. Zygophylloideae & Subfam. Zygophylloideae & 9 \\
\hline 8 & F. isotricha Murb. & Subfam. Zygophylloideae & Subfam. Zygophylloideae & Subfam. Zygophylloideae & 7 \\
\hline 9 & F. latifolia Delile & Subfam. Zygophylloideae & Subfam. Zygophylloideae & Subfam. Zygophylloideae & 7 \\
\hline 10 & F. mollis Delile & Subfam. Zygophylloideae & Subfam. Zygophylloideae & Subfam. Zygophylloideae & 8 \\
\hline 11 & F. scabra Forssk. & Subfam. Zygophylloideae & Subfam. Zygophylloideae & Subfam. Zygophylloideae & 10 \\
\hline 12 & F. sinaica Boiss & Subfam. Zygophylloideae & Subfam. Zygophylloideae & Subfam. Zygophylloideae & 5 \\
\hline 13 & F. tenuifolia Steud. \& Hochst. & Subfam. Zygophylloideae & Subfam. Zygophylloideae & Subfam. Zygophylloideae & 2 \\
\hline 14 & F. tristis Sickenb & Subfam. Zygophylloideae & Subfam. Zygophylloideae & Subfam. Zygophylloideae & 7 \\
\hline 15 & Peganum harmala $\mathrm{L}$. & Subfam. Peganoideae & Family Peganaceae & Family Peganaceae & 9 \\
\hline 16 & Seetzenia lanata (Willd.) Bullock & Subfam. Zygophylloideae & Subfam. Zygophylloideae & Subfam. Seetzenioideae & 3 \\
\hline 17 & Tetradiclis tenella Litv & Subfam. Tetradiclidoideae & Family Peganaceae & Family Peganaceae & 2 \\
\hline 18 & Tribulus bimucronatus Viv. & Subfam. Zygophylloideae & Family Tribulaceae & Subfam. Tribuloideae & 5 \\
\hline 19 & T. kaiseri Hosni & Subfam. Zygophylloideae & Family Tribulaceae & Subfam. Tribuloideae & 4 \\
\hline 20 & T. macropterus Boiss. & Subfam. Zygophylloideae & Family Tribulaceae & Subfam. Tribuloideae & 8 \\
\hline 21 & T. mollis Ehrenb. ex Schweinf. & Subfam. Zygophylloideae & Family Tribulaceae & Subfam. Tribuloideae & 4 \\
\hline 22 & T. pentandrus Forssk. & Subfam. Zygophylloideae & Family Tribulaceae & Subfam. Tribuloideae & 7 \\
\hline 23 & T. terrestris $\mathrm{L}$. & Subfam. Zygophylloideae & Family Tribulaceae & Subfam. Tribuloideae & 15 \\
\hline 24 & Zygophyllum aegyptium Hosny & Subfam. Zygophylloideae & Subfam. Zygophylloideae & Subfam. Zygophylloideae & 7 \\
\hline 25 & Z. album $\mathrm{L}$. & Subfam. Zygophylloideae & Subfam. Zygophylloideae & Subfam. Zygophylloideae & 15 \\
\hline 26 & Z. coccineum $\mathrm{L}$. & Subfam. Zygophylloideae & Subfam. Zygophylloideae & Subfam. Zygophylloideae & 20 \\
\hline 27 & Z. decumbens Delile & Subfam. Zygophylloideae & Subfam. Zygophylloideae & Subfam. Zygophylloideae & 7 \\
\hline 28 & Z. dumosum Boiss. & Subfam. Zygophylloideae & Subfam. Zygophylloideae & Subfam. Zygophylloideae & 2 \\
\hline 29 & Z. simplex $\mathrm{L}$. & Subfam. Zygophylloideae & Subfam. Zygophylloideae & Subfam. Zygophylloideae & 20 \\
\hline
\end{tabular}

STAND data, the procedures EIGEN, PROJ, and MXPLOT to perform the PCO. A PCO was preferred rather than a PCA (Principal Components Analysis), because a PCO performs better on data sets with missing data (Rohlf 1972).

\section{Results}

\section{Cluster analysis}

Figure 1 shows the UPGMA phenogram comprising all OTU's in the present study. The cophenetic correlation of distance matrix and tree matrix was 0.88 , indicating a good fit of the phenogram to the distance matrix (see Rohlf 1993).

Six branches and clusters can be distinguished: (1) a branch with Seetzenia lanata (subfamily Seetzenioideae).
This branch shows the largest distance from all other branches. (2) A cluster consisting of OUT's of the all species of Tribulus (subfamily Tribuloideae), (3) branch with Tetradiclis tenella (subfamily Tetradiclidoideae), (4) a branch with Balanites aegyptiacus (subfamily Balanitoideae), (5) a cluster which is divided into two subgroups: Peganum harmala (subfamily Peganoideae) and Zygophyllum coccineum (subfamily Zygophylloideae) subgroup, and a subgroup with all species of Zygophyllum (subfamily Zygophylloideae), and (6) a cluster comprising all species of Fagonia (subfamily Zygophylloideae).

\section{Principal coordinates analysis (PCO)}

The plot of 29 OTU's on the first three principal coordinates axes is shown in Figs. 2, 3, \& 4. These axes explain 
Table 2. Characters and character states used in morphometric analysis of the Zygophyllace

\begin{tabular}{|c|c|c|c|}
\hline $\mathrm{N}$ & Character & Character state & Code \\
\hline 1 & Life cycle & $\begin{array}{c}\text { Annual } \\
\text { annual or short lived perennial } \\
\text { Perennial } \\
\text { Perennial or sub shrub } \\
\text { Sub shrub } \\
\text { Shrub or tree }\end{array}$ & $\begin{array}{l}1 \\
2 \\
3 \\
4 \\
5 \\
6\end{array}$ \\
\hline 2 & \multicolumn{3}{|c|}{ Plant length (mean length in $\mathrm{cm}$ ) } \\
\hline 3 & Plant nature & $\begin{array}{c}\text { Prostrate } \\
\text { Decumbent to ascending } \\
\text { Ascending } \\
\text { Procumbent } \\
\text { Erect }\end{array}$ & $\begin{array}{l}1 \\
2 \\
3 \\
4 \\
5\end{array}$ \\
\hline 4 & Plant surface & $\begin{array}{c}\text { Glabrous } \\
\text { Glabrous to sparsely hairy } \\
\text { Sparsely hairy } \\
\text { Sparsely to densely hairy } \\
\text { Hairy }\end{array}$ & $\begin{array}{l}1 \\
2 \\
3 \\
4 \\
5\end{array}$ \\
\hline 5 & Hair shapes & $\begin{array}{c}\text { Simple } \\
\text { Glandular } \\
\text { Simple\stellate }\end{array}$ & $\begin{array}{l}1 \\
2 \\
3\end{array}$ \\
\hline 6 & Trichome stalk & $\begin{array}{l}\text { Present } \\
\text { Absent }\end{array}$ & $\begin{array}{l}1 \\
2\end{array}$ \\
\hline 7 & Stem nature & $\begin{array}{l}\text { Herbaceous } \\
\text { Woody at the base } \\
\text { Woody }\end{array}$ & $\begin{array}{l}1 \\
2 \\
3\end{array}$ \\
\hline 8 & Stem outline shape & $\begin{array}{c}\text { Terete } \\
\text { Quadrangular }\end{array}$ & $\begin{array}{l}1 \\
2\end{array}$ \\
\hline 9 & Nature of branches & $\begin{array}{c}\text { Spinescent } \\
\text { Not spinescent }\end{array}$ & $\begin{array}{l}1 \\
2\end{array}$ \\
\hline 10 & Stipules & $\begin{array}{l}\text { Present } \\
\text { Absent }\end{array}$ & $\begin{array}{l}1 \\
2\end{array}$ \\
\hline 11 & Stipules nature & $\begin{array}{l}\text { Spinescent } \\
\text { Membranous } \\
\text { Scarious } \\
\text { Bristle }\end{array}$ & $\begin{array}{l}1 \\
2 \\
3 \\
4\end{array}$ \\
\hline 12 & & Stipule length (mean length in $\mathrm{mm}$ ) & \\
\hline & & Leaf character & \\
\hline 13 & Leaves\leaflets arrangement & $\begin{array}{c}\text { Opposite } \\
\text { Alternate } \\
\text { Opposite below and alternate above }\end{array}$ & $\begin{array}{l}1 \\
2 \\
3\end{array}$ \\
\hline 14 & Leaf-bladelleaflets & $\begin{array}{l}\text { Flattened } \\
\text { Terete }\end{array}$ & $\begin{array}{l}1 \\
2\end{array}$ \\
\hline 15 & Leaf structure & $\begin{array}{c}\text { Simple or 1-foliolate } \\
\text { 2-foliolate } \\
\text { 1,3-foliolate } \\
\text { 3-foliolate } \\
\text { Multi-foliolate } \\
\text { Pinnatisect }\end{array}$ & $\begin{array}{l}1 \\
2 \\
3 \\
4 \\
5\end{array}$ \\
\hline
\end{tabular}

\begin{tabular}{|c|c|c|c|}
\hline \multirow{8}{*}{17} & \multirow{8}{*}{ Leafleaflet shape } & Linear & 1 \\
\hline & & Oblong to lanceolate & 2 \\
\hline & & Linear to elliptical & 3 \\
\hline & & Cylindrical & 4 \\
\hline & & Oblong to elliptical & 5 \\
\hline & & Ovate to elliptical & 6 \\
\hline & & Ovate & 7 \\
\hline & & Obovate & 8 \\
\hline
\end{tabular}


Table 2. Continuation

\begin{tabular}{llcc}
\hline $\mathrm{N}$ & Character & Character state & Code \\
\hline & & Mucronate & 1 \\
18 & Leaf apex & Acute & 2 \\
& & Obtuse & 3 \\
& & Retuse & 4 \\
\hline \multirow{2}{*}{19} & Petiolule & Present & 1 \\
& & Absent & 2 \\
\hline
\end{tabular}

Flower characters

\begin{tabular}{|c|c|}
\hline 20 & Flower pedicle (mean length in $\mathrm{mm}$ ) \\
\hline 21 & Sepal length (mean length in $\mathrm{mm}$ ) \\
\hline 22 & Sepal width (mean width in $\mathrm{mm}$ ) \\
\hline
\end{tabular}

\begin{tabular}{|c|c|c|c|}
\hline 23 & Sepal shape & $\begin{array}{c}\text { Lanceolate } \\
\text { Lanceolate to ovate } \\
\text { Ovate to oblong } \\
\text { Oblong } \\
\text { Obovate } \\
\text { Ovate }\end{array}$ & $\begin{array}{l}1 \\
2 \\
3 \\
4 \\
5 \\
6\end{array}$ \\
\hline 24 & Sepal apex & $\begin{array}{l}\text { Apiculate } \\
\text { Acute } \\
\text { Obtuse }\end{array}$ & $\begin{array}{l}1 \\
2 \\
3\end{array}$ \\
\hline 25 & Sepal surface & $\begin{array}{c}\text { Glabrous } \\
\text { Glabrous to sparsely hairy } \\
\text { Hairy }\end{array}$ & $\begin{array}{l}1 \\
2 \\
3\end{array}$ \\
\hline 26 & Sepal persistence at fruit maturity & $\begin{array}{l}\text { Persistent } \\
\text { Deciduous }\end{array}$ & $\begin{array}{l}1 \\
2\end{array}$ \\
\hline 27 & Petal Persistence & $\begin{array}{l}\text { Present } \\
\text { Absent }\end{array}$ & $\begin{array}{l}1 \\
2\end{array}$ \\
\hline 28 & & Petal length (mean length in $\mathrm{mm}$ ) & \\
\hline 29 & & Petal width (mean width in $\mathrm{mm}$ ) & \\
\hline 30 & Petal shape & $\begin{array}{c}\text { Obovate } \\
\text { Obovate -oblong } \\
\text { Spathulate } \\
\text { Spathulate with long claws }\end{array}$ & $\begin{array}{l}1 \\
2 \\
3 \\
4\end{array}$ \\
\hline 31 & Petal colour & $\begin{array}{c}\text { White } \\
\text { Cream } \\
\text { Yellow to white } \\
\text { Yellow } \\
\text { Pink } \\
\text { Pink to mauve } \\
\text { Violet }\end{array}$ & $\begin{array}{l}1 \\
2 \\
3 \\
4 \\
5 \\
6 \\
7\end{array}$ \\
\hline 32 & Petal apex & $\begin{array}{l}\text { Apiculate } \\
\text { Obtuse }\end{array}$ & $\begin{array}{l}1 \\
2\end{array}$ \\
\hline 33 & Stamens number & $\begin{array}{c}15 \\
10 \\
5-10 \\
5 \\
4\end{array}$ & $\begin{array}{l}1 \\
2 \\
3 \\
4 \\
5\end{array}$ \\
\hline 34 & & Filament length (mean length in $\mathrm{mm}$ ) & \\
\hline 35 & Filament appendages & $\begin{array}{c}\text { Present } \\
\text { Absent }\end{array}$ & $\begin{array}{l}1 \\
2\end{array}$ \\
\hline 36 & Ovary surface & $\begin{array}{c}\text { Glabrous } \\
\text { Glabrous to sparsely hairs } \\
\text { Hairy }\end{array}$ & $\begin{array}{l}1 \\
2 \\
3\end{array}$ \\
\hline 37 & Nectariferous disc below ovary & $\begin{array}{l}\text { Present } \\
\text { Absent }\end{array}$ & $\begin{array}{l}1 \\
2\end{array}$ \\
\hline
\end{tabular}


Table 2. Continuation

\begin{tabular}{|c|c|c|c|}
\hline $\mathrm{N}$ & Character & Character state & Code \\
\hline \multirow{2}{*}{38} & \multirow{2}{*}{ Style number } & 1 & 1 \\
\hline & & 5 & 2 \\
\hline \multirow[t]{2}{*}{39} & \multicolumn{2}{|r|}{ Style length (mean length in mm) } & \\
\hline & \multicolumn{3}{|c|}{ Pollen grain characters } \\
\hline \multirow{4}{*}{40} & \multirow{4}{*}{ Pollen shape } & Oblate spheroidal $(\mathrm{P} / \mathrm{E}=0.88-1)$ & 1 \\
\hline & & Spheroidal $(\mathrm{P} / \mathrm{E}=1)$ & 2 \\
\hline & & Subprolate $(\mathrm{P} / \mathrm{E}=1.14-1.33)$ & 3 \\
\hline & & Prolate $(\mathrm{P} / \mathrm{E}=1.33-2)$ & 4 \\
\hline \multirow{3}{*}{41} & \multirow{3}{*}{ Pollen types } & Tricolpate & 1 \\
\hline & & Tricolporate & 2 \\
\hline & & Pantoporate & 3 \\
\hline \multirow{6}{*}{42} & \multirow{6}{*}{ Exine sculpture } & Striate-perforate & 1 \\
\hline & & $\begin{array}{l}\text { strate-perrorate } \\
\text { Rugulate-reticulate }\end{array}$ & 2 \\
\hline & & Micro-reticulate & 3 \\
\hline & & Reticulate & 4 \\
\hline & & Coarsely-reticulate & 5 \\
\hline & & Fruit characters & \\
\hline \multirow{3}{*}{43} & \multirow{3}{*}{ Fruit type } & Loculicidal capsule & 1 \\
\hline & & Schizocarpic & 2 \\
\hline & & Drupe & 3 \\
\hline 44 & & Fruit length (mean length in $\mathrm{mm}$ ) & \\
\hline 45 & & Fruit width (mean width in $\mathrm{mm}$ ) & \\
\hline \multirow{3}{*}{46} & \multirow{3}{*}{ Fruit surface } & Glabrous & 1 \\
\hline & & Glabrous to sparsely hairs & 2 \\
\hline & & Hairy & 3 \\
\hline \multirow{2}{*}{47} & \multirow{2}{*}{ Fruit wings } & Winged & 1 \\
\hline & & Not Winged & 2 \\
\hline \multirow{7}{*}{48} & \multirow{7}{*}{ Fruit shape } & Obovate & 1 \\
\hline & & Obconical & 2 \\
\hline & & Oblong-ellipsoid & 3 \\
\hline & & Spherical & 4 \\
\hline & & Sub spherical & 5 \\
\hline & & Pyramidal & 6 \\
\hline & & Disc-shaped & 7 \\
\hline \multirow{3}{*}{49} & \multirow{3}{*}{ Mericarp edge } & Spiny & 1 \\
\hline & & Winged & 2 \\
\hline & & Not winged & 3 \\
\hline \multirow{3}{*}{50} & \multirow{3}{*}{ Number of fruit locules } & 3 & 1 \\
\hline & & 4 & 2 \\
\hline & & 5 & 3 \\
\hline 51 & & Fruit pedicel (mean length in $\mathrm{mm}$ ) & \\
\hline
\end{tabular}

Seed characters

\begin{tabular}{llcl}
\hline & & Ellipsoid & \\
& & Oblong & 2 \\
52 & Seed shape & Obovoid & 3 \\
& & Ovoid & 4 \\
& & Flat to ovoid & 5 \\
\hline & & Smooth & 1 \\
& Seed architecture & Tuberculate & 2 \\
& & Crustaceous & 3 \\
\hline
\end{tabular}


Table 2. Continuation

\begin{tabular}{|c|c|c|c|}
\hline $\mathrm{N}$ & Character & Character state & Code \\
\hline \multirow{4}{*}{54} & \multirow{4}{*}{$\begin{array}{l}\text { Seed size }(\mathrm{mm}) \\
\text { (Length } \mathrm{x} \text { width) }\end{array}$} & $0.6-1.7 \times 0.4-1.3$ & 1 \\
\hline & & $1.8-4.2 \times 1.3-3.7$ & 2 \\
\hline & & $4.3-24 \times 1.6-3$ & 3 \\
\hline & & $25-35 \times 10-15$ & 4 \\
\hline \multirow{2}{*}{55} & \multirow{2}{*}{ Endosperm of seed } & Present & 1 \\
\hline & & Absent & 2 \\
\hline \multirow{4}{*}{56} & \multirow{4}{*}{ Epidermal cell patterns } & Isodiametric or 4-5-6 polygonal & 1 \\
\hline & & Irregular or 4-5-6 polygonal & 2 \\
\hline & & Irregular or polygonal cells & 3 \\
\hline & & 4-5 gonals & 4 \\
\hline \multirow{2}{*}{57} & \multirow{2}{*}{ Anticlinal walls } & Straight & 1 \\
\hline & & Straight to slightly sinuous & 2 \\
\hline \multirow{3}{*}{58} & \multirow{3}{*}{ Relief of cell wall boundaries } & Raised & 1 \\
\hline & & Raised-channeled & 2 \\
\hline & & Channeled & 3 \\
\hline \multirow{3}{*}{59} & \multirow{3}{*}{ Sculpture of anticlinal boundaries } & Smooth & 1 \\
\hline & & Smooth to fine folded & 2 \\
\hline & & Folded & 3 \\
\hline \multirow{5}{*}{60} & \multirow{5}{*}{ Curvature of outer periclinal cell wall } & & 1 \\
\hline & & $\begin{array}{l}\text { Flat to concave } \\
\text { Flat to convex }\end{array}$ & 2 \\
\hline & & Flat to concave with sunken central papilla & 3 \\
\hline & & Convex & 3 \\
\hline & & Domate with globular central papilla & $\begin{array}{l}4 \\
5\end{array}$ \\
\hline \multirow{6}{*}{61} & \multirow{6}{*}{ Sculpture of periclinal cell wall } & Smooth & 1 \\
\hline & & Smooth to fine folded & 2 \\
\hline & & Folded & 3 \\
\hline & & Radiate-striate & 4 \\
\hline & & Micro-reticulate & 5 \\
\hline & & Reticulate & 6 \\
\hline
\end{tabular}

$68.83 \%$ of the total observed variation. Plots $1 / 2,1 / 3$, and $2 / 3$ together show six groups. On the first axis $(31.60 \%$ of the total variation, Figs $2 \& 3$ ), a separation is demonstrated between four groups:- 1) group of all species of Tribulus (subfamily Tribuloideae); 2) group comprising all species of Fagonia (subfamily Zygophylloideae; 3) Seetzenia lanata (subfamily Seetzenioideae); 4) group of Peganum harmala (subfamily Peganoideae) nested within the genus Zygophyllum (subfamily Zygophylloideae). The main characters explaining this separation (characters with high factor loading $>0.6$ ) were fruit shape, seed architecture, mericarp edge, leaf petiole length, seed shape, filament length, seed size, epidermal cell patterns, curvature of outer periclinal cell wall, pollen shape, relief of cell wall boundaries, fruit width, leaf structure and hair shapes (Table 3). The second principal coordinates axis ( $19.96 \%$ of the total variation, Figs. 2 \& 4) reveals a split between two groups: 1) Balanites aegyptiacus (subfamily Balanitoideae) and 2) Tetradiclis tenella (subfamily Tetradiclidoideae). This split was based mainly on stipules nature, pollen types, pollen shape, seed architecture, stipules length, petal width, curvature of outer periclinal cell wall, sepal length, and petal colour (Table 3). Along the third axis (17.27\% of the total variation, Figs. 3 \& 4) a separation between group of Peganum harmala (subfamily Peganoideae) nested within the genus
Zygophyllum (subfamily Zygophylloideae), based on flower pedicle length, fruit type, stamens number, plant nature, sepal shape, sculpture of anticlinal boundaries, style length, number of fruit locules, plant cycle, leaf structure, sculpture of periclinal cell wall, leaf/leaflet shape, leaf apex, pollen types, and petal length.

\section{Discussion}

Systematics must largely rely on morphological characters to define taxa. Problems in classification arise when the taxa display a large amount of variability, due to phenotypic plasticity (van den Berg \& Groendijk-Wilders 1999). Groups within the Zygophyllaceae have become specialized for similar arid habitats, which has probably led to much parallel and convergent evolution. Some characters which turn out to be among the most homoplasious and distinguish taxa, such as the presence of staminal appendages, nectariferous disc and endosperm. These characters may be of value only among subgroups within the family and for distinguishing genera, and not for phylogenetic purposes in the whole family (Sheahan \& Chase 1996). Several authors have tried to provide a natural system to divide the Zygophyllaceae into subfamilies, tribes and sections (Engler 1896a; 1931; 


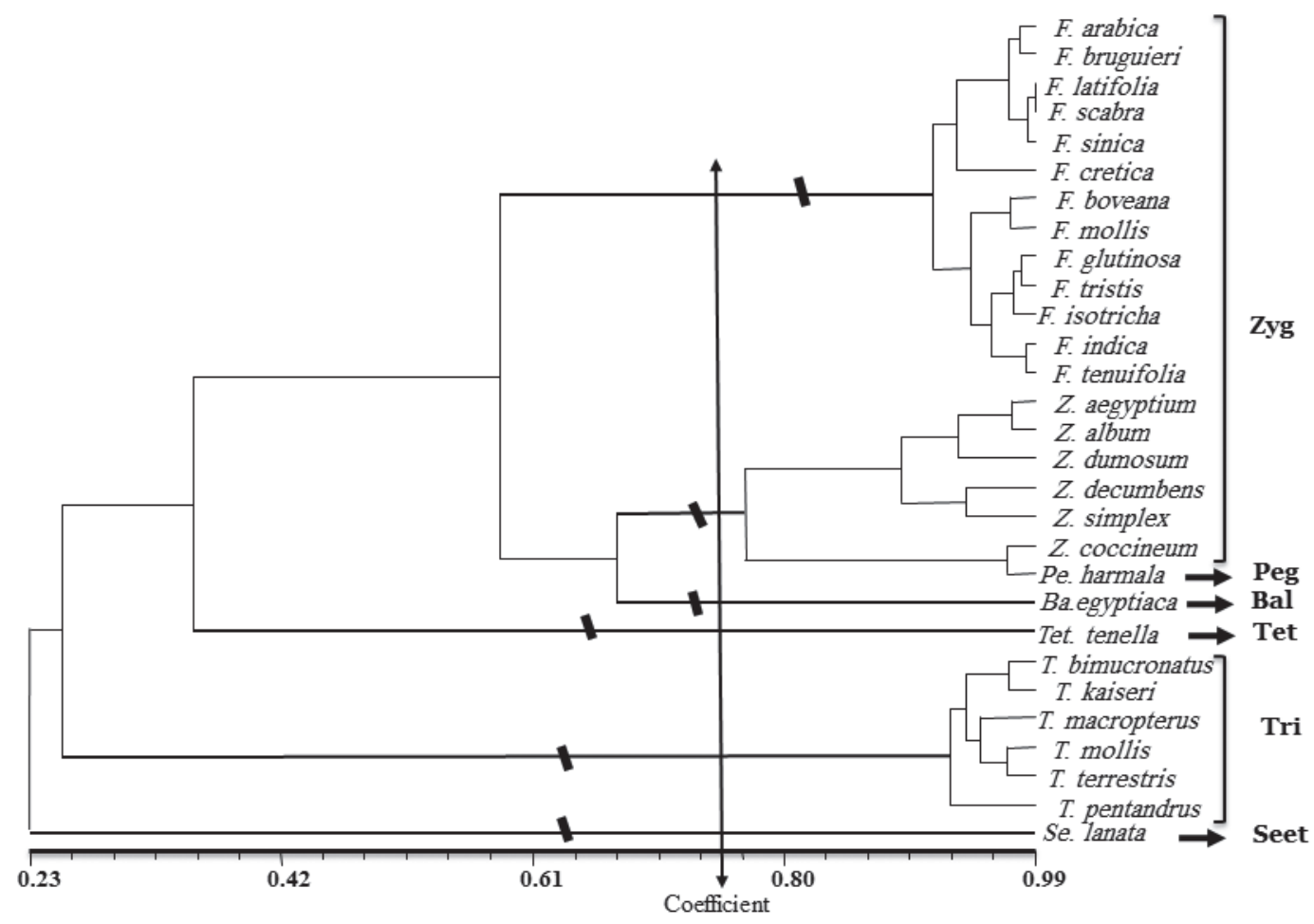

Figure 1. Phenogram of the 29 studied taxa, clustering with UPGMA method: Bal, Balanitoideae; Peg, Peganoideae; Seet, Seetzenioideae; Tet, Tetradiclidoideae; Tri, Tribuloideae; Zyg, Zygophylloideae.

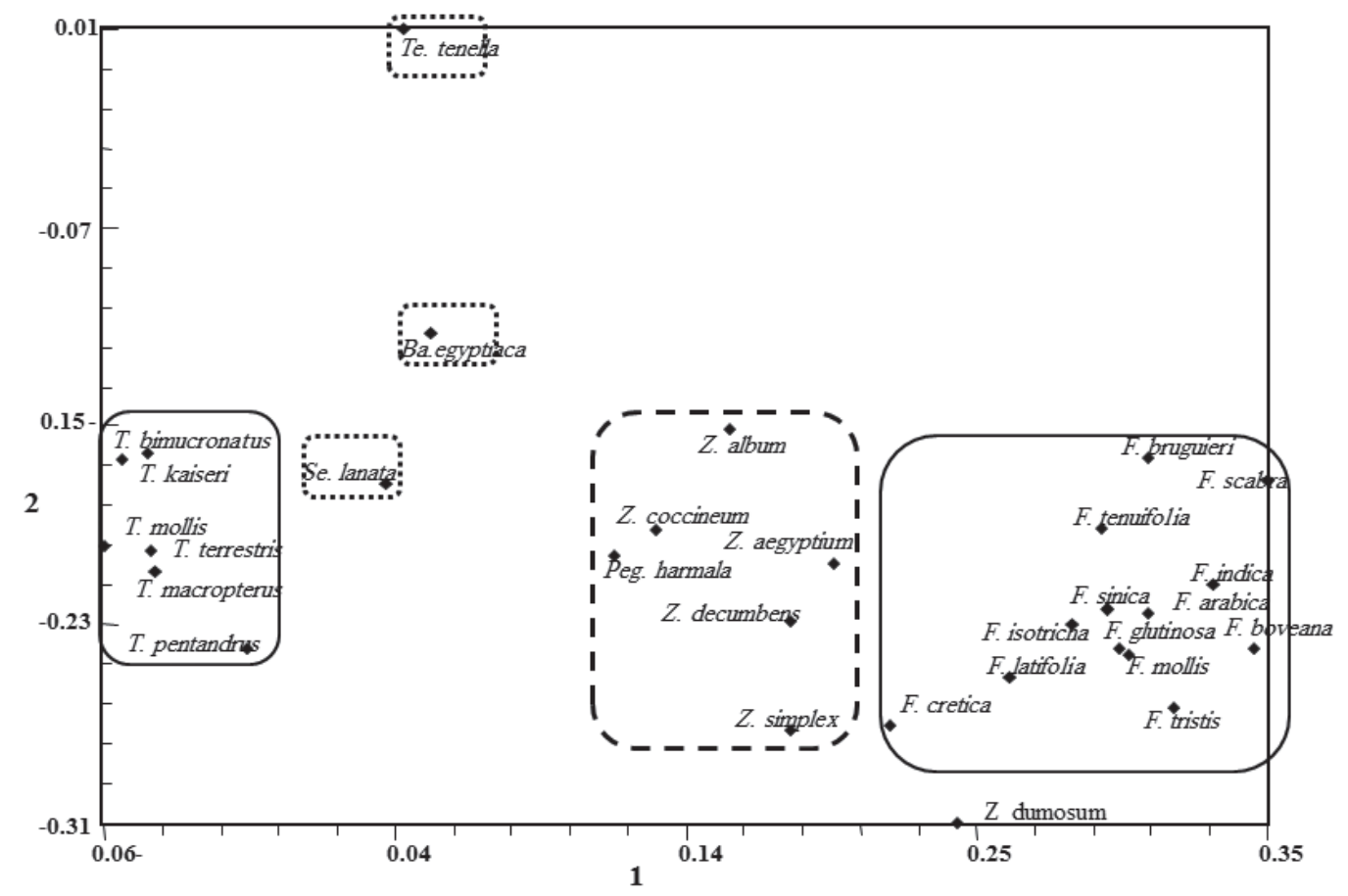

Figure 2. Scatterplot of the 29 OUTs plotted against the first principal coordinate by the second principal coordinate. 


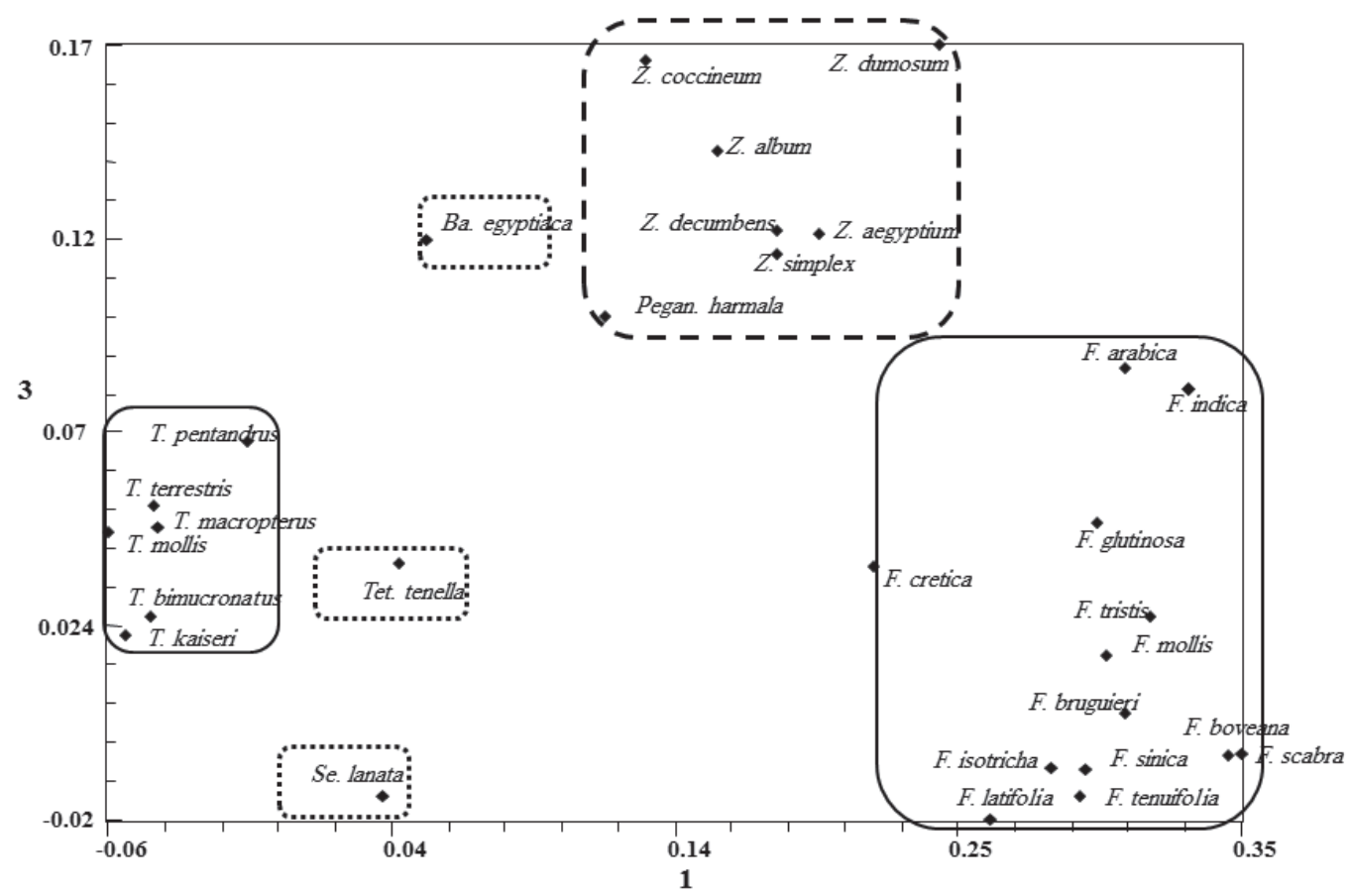

Figure 3. Scatterplot of the 29 OUTs plotted against the first principal coordinate by the third principal coordinate.

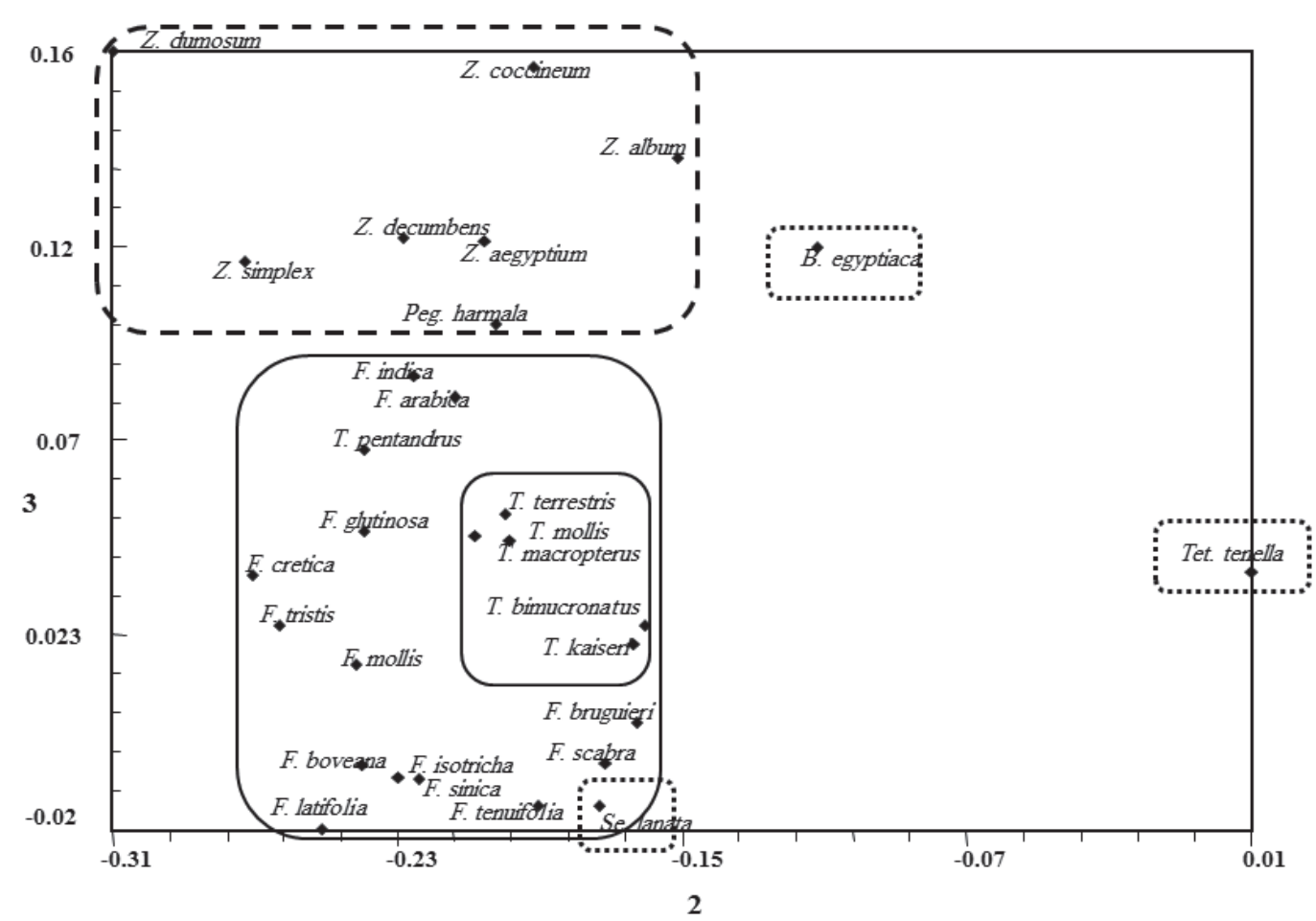

Figure 4. Scatterplot of the 29 OUTs plotted against the second principal coordinate by the third principal coordinate. 
Table 3. Morphological characters showing highest factor loading on the first three Principal coordinate axes. The shaded numbers mean characters with high factor loading $>0.6$.

\begin{tabular}{|c|c|c|c|c|}
\hline \multirow{3}{*}{$\mathrm{N}$} & \multirow{3}{*}{ Characters } & \multicolumn{3}{|c|}{ Principal coordinates } \\
\hline & & 1 & 2 & 3 \\
\hline & & \multicolumn{3}{|c|}{ Factors loading } \\
\hline 1 & Plant cycle & 0.56 & 0.12 & 0.76 \\
\hline 2 & Plant length (mean length in $\mathrm{cm}$ ) & -0.13 & -0.51 & 0.55 \\
\hline 3 & Plant nature & 0.31 & -0.24 & -0.95 \\
\hline 4 & Plant surface & 0.24 & 0.12 & -0.13 \\
\hline 5 & Hair shapes & 0.60 & -0.41 & -0.42 \\
\hline 6 & Trichome stalk & -0.30 & 0.23 & -0.28 \\
\hline 7 & Stem nature & 0.21 & 0.27 & 0.20 \\
\hline 8 & Stipules nature & -0.18 & -0.99 & -0.27 \\
\hline 9 & Stipules length (mean length in $\mathrm{mm}$ ) & 0.26 & -0.71 & -0.29 \\
\hline 10 & Leaves lleaflets arrangements & 0.23 & 0.50 & 0.40 \\
\hline 11 & Leaf structure & -0.61 & -0.17 & -0.70 \\
\hline 12 & Leaf petiole measurements (mean length in $\mathrm{cm}$ ) & 0.86 & 0.29 & 0.44 \\
\hline 13 & Leafleaflet shape & -0.38 & -0.28 & -0.65 \\
\hline 14 & Leaf apex & -0.24 & -0.16 & -0.65 \\
\hline 15 & Flower pedicle (mean length in $\mathrm{mm}$ ) & -0.38 & -0.18 & 0.96 \\
\hline 16 & Sepal length (mean length in mm) & -0.11 & -0.63 & 0.42 \\
\hline 17 & Sepal width (mean width in $\mathrm{mm}$ ) & 0.19 & -0.50 & 0.52 \\
\hline 18 & Sepal shape & -0.10 & -0.18 & 0.94 \\
\hline 19 & Sepal apex & 0.38 & 0.24 & 0.46 \\
\hline 20 & Sepal surface & -0.24 & 0.20 & 0.43 \\
\hline 21 & Petal length (mean length in $\mathrm{mm}$ ) & 0.26 & -0.54 & -0.61 \\
\hline 22 & Petal width (mean width in $\mathrm{mm}$ ) & 0.20 & -0.67 & 0.41 \\
\hline 23 & Petal shape & 0.27 & -0.22 & 0.36 \\
\hline 24 & Petal colour & 0.28 & 0.63 & 0.51 \\
\hline 25 & Petal apex & 0.37 & -0.25 & 0.38 \\
\hline 26 & Stamens number & 0.35 & 0.12 & 0.95 \\
\hline 27 & Filament length (mean length in $\mathrm{mm}$ ) & -0.80 & -0.17 & -0.40 \\
\hline 28 & Ovary surface & 0.45 & -0.41 & 0.38 \\
\hline 29 & Style length (mean length in $\mathrm{mm}$ ) & -0.22 & -0.19 & -0.93 \\
\hline 30 & Pollen shape & 0.73 & -0.81 & 0.35 \\
\hline 31 & Pollen types & 0.31 & -0.91 & -0.63 \\
\hline 32 & Exine sculpture & 0.31 & -0.10 & -0.54 \\
\hline 33 & Fruit type & 0.51 & 0.41 & 0.95 \\
\hline 34 & Fruit length (mean length in $\mathrm{mm}$ ) & -0.33 & -0.59 & 0.21 \\
\hline 35 & Fruit width (mean width in $\mathrm{mm}$ ) & -0.63 & -0.40 & 0.29 \\
\hline 36 & Fruit surface & -0.12 & -0.26 & -0.40 \\
\hline 37 & Fruit wings & -0.16 & -0.17 & -0.21 \\
\hline 38 & Fruit shape & -0.95 & -0.45 & 0.33 \\
\hline 39 & Mericarp edge & -0.88 & -0.32 & -0.26 \\
\hline 40 & Number of fruit locules & 0.49 & 0.13 & 0.91 \\
\hline 41 & Fruit pedicel (mean length in mm & -0.54 & -0.50 & 0.22 \\
\hline 42 & Seed shape & 0.83 & 0.21 & -0.16 \\
\hline 43 & Seed architecture & 0.91 & 0.72 & 0.23 \\
\hline 44 & Seed size in $\mathrm{mm}$ (Length $\mathrm{x}$ width) & 0.79 & 0.26 & 0.30 \\
\hline 45 & Epidermal cell paterns & 0.78 & -0.13 & -0.58 \\
\hline 46 & Anticlinal walls & 0.15 & 0.56 & 0.18 \\
\hline 47 & Relief of cell wall boundaries & 0.66 & -0.13 & -0.45 \\
\hline 48 & Sculpture of anticlinal boundaries & 0.10 & -0.12 & 0.94 \\
\hline 49 & Curvature of outer periclinal cell wall & 0.74 & -0.66 & -0.46 \\
\hline 50 & Sculpture of periclinal cell wall & 0.10 & -0.13 & 0.67 \\
\hline \multicolumn{2}{|c|}{ Percentage per PCO } & 31.60 & 19.96 & 17.27 \\
\hline Perc & e first three principal coordinates amount 68.83 & & & \\
\hline
\end{tabular}


El Hadidi 1975; Sheahan \& Chase 1996; 2000; Beier et al. 2003, see Table 1). Some of these studies were based on a small number of morphological characters or few numbers of the taxa. In the present study, a large number of characters were scored and numerical methods (UPGMA and PCO) were applied to study the relationships between taxa and to estimate the level of variation between them. UPGMA gives insight into the degree of similarity among the OTUs and whether they form groups/ clusters, and indicates the level of variation between species. On the other hand, $\mathrm{PCO}$ reflects which characters are important on the axes, and indicates the significant characters on the basis of the highest factor loading (Table 3). Thus it becomes clear which characters help differentiate between the groups and can be useful to distinguish taxa. Generally, our results demonstrated similarity between the UPGMA clustering and PCO ordination. In general, six taxonomic groups can be distinguished

\section{Fagonia group}

The taxonomy of Fagonia is very difficult mainly due to a high degree of plasticity and thereby adaptations to climatic conditions (Zohary 1972; Danin 1996). Ozenda \& Quézel (1956) grouped the North African Fagonia species into four natural groups: (1) the F. kahirina-cretica-flamandii group, (2) the F. arabica-bruguieri group, (3) the F. glutinosa-latifolia group, and (4) the F. microphylla- group. However, El Hadidi (1966) classified Fagonia species into three natural groups based on pollen and trichome morphology. Also, El Hadidi (1972 \& 1974) classified Fagonia-species into complexes. Beier et al. (2004) studied the phylogenetic relationships within genus Fagonia based on trnL \& ITS DNA sequences and they did not support the natural groups of Fagonia, and showed that all species from the Old World, except Fagonia cretica form a weakly supported clade. Abdel Khalik \& Hassan (2012) investigated seed and trichome morphology of Fagonia in Egypt and indicated that the seed and trichome morphology are useful in distinguishing the species and not supporting the natural groups. The results of both cluster and principal coordinated analysis presented here confirmed that all species of Fagonia form a well-distinguished group, characterized by several characteristics: obconical fruit shape, smooth seed architecture, non-winged mericarp edge, ovoid seed shape, filament length $(3-7 \mathrm{~mm})$, seed size (1.8-4.2 x 1.3-3.7 mm), subprolate to prolate pollen shape, 1-3-foliolate leaf and glandular hair shapes. Within this group, we can show that Fagonia arabica, F. bruguieri, F. sinaica, F. latifolia, and F. scabra form a subgroup; F. cretica forms a subgroup and another one includes the remaining species of Fagonia. These results are incongruent with those of Ozenda \& Quézel (1956), El Hadidi (1966; 1972; 1974) and partially agree with both of Beier et al. (2004) and Abdel Khalik \& Hassan (2012).

\section{Zygophyllum and Peganum group}

Van Zyl (2000) presented morphological analysis of the genus Zygophyllum in South Africa, and supported the divi- sion of Zygophyllum into the two subgenera Agrophyllum and Zygophyllum, which was contended by previous authors (Endlicher 1841; Van Huyssteen 1937). Van Huyssteen (1937) treated Z. coccinum, Z. album and Z. aegyptium as members of section Mediterranea Engl., and circumscribed Z. simplex and $Z$. decumbens in the section Bipartia Huysst. Moreover, she placed $Z$. dumosum in a separate section Alata Huysst. based on characters of fruit, stamens, and leaves characters. Sheahan \& Chase (2000) analysed both $r b c \mathrm{~L}$ and $\operatorname{trnL}$-F sequences from 36 members of Zygophyllaceae and indicated that Zygophyllum is a polyphyletic genus, and show that, both $Z$. simplex and $Z$. decumbens were joined together in the same clade and the clade is a sister to the clade composed of Zygophyllum album, Z. coccinum, and Tetraena.

In some publications Peganum was placed in a separate family (Takhtajan 1969; El Hadidi 1975). Furthermore, Sheahan \& Chase (1996) divided Zygophyllaceae into five subfamilies, whereas Peganum, Malacocarpus and Nitraria were separated from Zygophyllaceae and close to members of Sapindales, and also they recommended of Peganaceae and Nitrariaceae. However, Takhtajan (1980) and El Hadidi \& Fayed (1995) returned it to Zygophyllaceae. The results of both cluster and principal coordinated analysis confirmed that the group of all species of Zygophyllum and Peganum harmala is a well-distinguished group, characterized by: short flower pedicle length (1-7 mm), schizocarpic fruits, 10 stamens, style length (1-2 mm), perennial to sub shrub plants, 2 -foliolates, obtuse leaf apex, tricolpate pollen type, 5 locules per fruit. Within this group, we can show three subgroups: Zygophyllum simplex and Z. decumbens subgroup; $Z$. album, $Z$. aegyptium and $Z$. dumosum subgroup, and another subgroup includes $Z$. coccineum and Peganum harmala. Our results identify three branches that are congruent with the Zygophyllum sections proposed by Van Huyssteen (1937), and the results from previous studies of phylogenetic relationships by Sheahan \& Chase (2000) and Beier et al. (2003). Moreover, the results indicate that Peganum harmala is not such an isolated species as has been thought (Takhtajan 1969; El Hadidi 1975; Sheahan \& Chase 1996). Some characters that have been put advance as unique for it, such as tricolporate pollen grains, non-winged fruit, 5 styles, present of nectiferous disc, and endospermic seed, are also found in the other members of Zygophyllum, and these results are congruent with those of Takhtajan (1980) and El Hadidi \& Fayed (1995).

\section{Tetradiclis group}

Engler (1896a; 1931) delimited Tetradiclis under subfamily Tetradiclidoideae with in Zygophyllaceae. However, El Hadidi (1975); Takhtajan (1980, 1983); Sheahan \& Chase $(1996 ; 2000)$, and Bolous (2000) removed it from the family Zygophyllaceae and put it under a family Peganaceae. Moreover, Takhtajan (1996) treats it under a separate monotypic family Tetradiclidaceae. Morphologically, Tetradiclis tenella is quite distinct and easily identifiable. It is distinguished 
from other Zygophyllaceae by the exstipulate pinnatisect leaves, , flower small $(1 \mathrm{~mm})$ and tetramerous, 4 loculed fruit. It shares with genera of Zygophyllaceae the absence of filament appendages, the annual habit, the loculicidal capsule, flattened leaf blade, and the obtuse leaf apex. The results of both cluster and principal coordinates analyses distinct the group from the other species by herbaceous stem, exstipulate leaves, leaves opposite below and alternate above, tetramerous flower, 4 loculed fruit.

\section{Balanites group}

Based on the morphological and anatomical studies within the Balanites, it was suggested that the genus can be assigned circumscribed within its own family Balanitaceae (Hutchinson 1973; El Hadidi 1975; Takhtajan 1983; Sands 1989). However, Engler (1896a;1931); Scholz (1964); Hegnauer (1973); Cronquist (1981), and Sheahan \& Chase (1996; 2000) included it in Zygophyllaceae. In my results, both cluster and principal coordinates analysis, Balanites aegyptiacus is separated from other groups, and characterized by shrub to tree habit, spinescent branches, exstipulate leaves, striate-perforate pollen sculpture, drupaceous fruit, and crustaceous seed. It is close to members of $\mathrm{Zy}$ gophylloideae and Peganoideae based mainly on stamen numbers (10), tricolporate pollen type, 5 loculed-fruit. From the results of this study it appears that Balanites (subfamily Balanitoideae) is closed to the members of Zygophyllaceae and embedded within it. Consequently, it should not be regarded as a separate family and this result agrees with those of Engler (1896a; 1931); Scholz (1964); Hegnauer (1973); Cronquist (1981), and Sheahan \& Chase (1996; 2000).

\section{Tribulus group}

Engler (1931) put Tribulus in tribe Tribuleae within the subfamily Zygophylloideae. However, El-Hadidi (1975; 1977) proposed a new family Tribulaceae based on Engler's tribe Tribulae. El Hadidi (1978) recognized three sections in Tribulus, each with a specific diagnostic character: (1) section Terrestris L. (T. terrestris, T. parvispinus \& T. spurius) with spiny mericarps, (2) section Alata Hadidi (T. macropterus, T. megistopterus \& T. pentandrus) with winged mericarps and (3) section Inermis Hadidi (T. mollis, T. kaiseri \& T. bimucronatus) with spineless or wingless mericarps. According to Hosni (1978), the most reliable and constant characters for Tribulus species, and hence of major systematic value, are those of the mature carpels and the size of the flower, while other characters such as the habit of the plant, hairiness, size of leaf, morphology of the style and stigma are of minor systematic value. Furthermore, Mohamed (2006) studied seed morphology, seed proteins and Iso-enzymes in Tribulus, and divided this genus into two main groups: (i) T. macropterus and T. mollis, and (ii) T. terrestris, T. bimucronatus, T. pentandrus, and T. kaiseri. The results of both cluster and principal coordinated analysis confirmed that the group of all species of Tribulus is a well-distinguished group, characterized by: multi- foliolate leaf, opposite leaflet, flat, 5-10 stamens, filament without appendages, oblate spheroidal pollen shape, pantoporate, coarsely-reticulate pollen schizocarpic fruit, 5 indehiscent mericarps. Within this group, we can show that T. bimucronatus and T. kaiseri form two subgroups: 1) T. macropterus, T. mollis, and T. terrestris and 2) another one includes T. pentandrus. These results disagree with El Hadidi (1978) and partially agree with Mohamed (2006).

\section{Seetzenia group}

Engler (1931) and El Hadidi (1975) placed Seetzenia with Fagonia and Zygophyllum in the subfamily Zygophylloideae within the family Zygophyllaceae. However, Sheahan \& Chase $(1996 ; 2000)$ proposed a new subfamily Seetzenioideae. According to the cluster and principal coordinates analysis, Seetzenia lanata (Seetzenioideae) shows the largest distance from all other groups, and is distinct from the others by trifoliate leaves, apetalous flowers, 5 stamens, 5 styles, tricolpate pollen type, oblong seed shape, crustaceous seed architecture. This result disagree with those of Engler (1931) and El Hadidi (1975) and agrees with Sheahan \& Chase $(1996 ; 2000)$ for separating it as a subfamily.

\section{Conclusion}

UPGMA and PCO analysis can be used to study the morphological variation within the family to determine the discontinuities among genera and subfamilies. My results indicate that Zygophyllaceae are heterogeneous, including Peganum harmala which has been proposed to form a separate family. There are many splits between subfamilies Zygophylloideae, Tribuloideae, Tetradiclidoideae and Seetzenioideae which seem to be distinct groups. However, there is also some degree of similarity among certain taxa of the subfamilies Balanitoideae, Peganoideae and Zygophylloideae. I consider Zygophyllum the most heterogeneous of the Zygophylloideae because I found that the taxa from this genus interspersed with taxa from Peganoideae (Peganum harmala), and this is congruent with the results of Sheahan \& Chase (2000). Although this study has contributed new conclusions to literature, it is limited to the known genera in Egypt. A comprehensive study covering all genera would be necessary to make a more thorough classification and it would be very useful for the further studies to use molecular data.

\section{Acknowledgements}

I am grateful to the Director and Curator of Cairo University Herbarium (CAI) and Agriculture Museum in Dokki (CAIM). My great thanks are due to Prof. dr. van der Maesen, Professor of Plant Taxonomy in Biosyste- 
matics group, Plant Science, Wageningen University, the Netherlands for going through the manuscript and making valuable suggestions.

\section{References}

Abdel Khalik, K. \& Hassan, N.M.S. 2012. Seed and trichome morphology of the Egyptian Fagonia (Zygophyllaceae) with emphasis on their systematic implications. Nordic Journal of Botany 30(1): 116-126.

APG (Angiosperm Phylogeny Group) 2009. An update of the Angiosperm Phylogeny Group classification for the orders and families of flowering plants.: APG III. Botanical Journal of the Linnean Society161: 105-121.

Agababyan, V.S. 1964. Morphological types of pollen and systematic classification of the Zygophyllaceae. Izvestiya Akademii Nauk Armyanskoi, SSR, Biologicheskikh Nauk 17: 39-45.

Beier, B.A.; Chase, M.W. \& Thulin, M. 2003. Phylogenetic relationships and taxomony of subfamily Zygophylloideae (Zygophyllaceae) based on molecular and morphological data. Plant Systematic and Evolution 240: 11-39.

Boulos, L. 2000. Flora of Egypt. Vol. 2. Cairo, Al Hadara Publishing.

Cronquist, A. 1968. The evolution and classification of flowering plants. London, Thomas Nelson.

Cronquist, A. 1981. An integrated system of classification of flowering plants. New York, Columbia University Press.

Danin, A. 1996. Plants of Desert Dunes. Berlin-Heidelberg, Springer.

El Hadidi, M.N. 1966. The genus Fagonia in Egypt. Candollea 21: 13-54.

El Hadidi, M.N. 1972. The family Zygophyllaceae in Egypt. Botaniska Notiser 125: 523-534.

El Hadidi, M.N. 1974. Weitere Beobachtungen an der Gattung Fagonia. Mitteilungen der Botanischen Staatssammlung München 11: 387.

El Hadidi, M.N. 1975. Zygophyllaceae in Africa. Boissieria 24: 317-323.

El Hadidi, M.N. 1977. Two new Zygophyllum species from Arabia. Publication of Cairo University Herbarium 7/8: 327-331.

El Hadidi, M.N. 1978. Zygophyllaceae. Webbia 33: 45-101.

El Hadidi, M.N. \& Fayed, A.A. 1995. Materials for an? Excursion Flora of Egypt. Taeckholmia 15: 40-53.

Endlicher, S.L. 1841. Genera plantarum. Part 18: 1161. Fr. Beck, Vienna.

Engler, A. 1896a. Zygophyllaceae. In: Engler A, Prantl K, eds. Die nat"urliche Pflanzenfamilien, 1st edition, vol. 3, Section 4: 74-93. Leipzig, Engelmann.

Engler, A. 1931. Zygophyllaceae. In: Engler A, Prantl K, eds. Die natürliche Pflanzenfamilien, 2nd edition, vol. 19a, section 2: 144-184. Leipzig, Engelmann.

Erdtman, G. 1952. Pollen Morphology and Plant Taxonomy. Angiosperms. Waltham, Chronica Botanica Co.

Hegnauer, R. 1973. Chemotaxonomie der Pflanzen. Basle and Stuttgart, Birkhauser.

Hosni, H.A. 1978. Revision of the genus Tribulus L., sections Alata and Inermis in Egypt and Arabia. M.Sc. Thesis, Faculty of Science, Cairo University.

Hutchinson, J. 1973. The families of flowering plants. Oxford, Clarendon Press.

Kuprianova, L.A. \& Alyoshina, L.A. 1978. Pollen dicotyledoneaerum Florae Partis Europareae. URSS. Lamiaceae-Zygophyllaceae.(in Russian). Nauka 184 p. Komarov Botanical Institute of Russian Academy of Sciences

Mohamed, A.H. 2006. Taxonomic Significance of Seed Proteins and Iso- enzymes in Tribulus (Zygophyllaceae). International Journal of Agriculture \& Biology 8(5): 573-575.
Ozenda, P. \& Quézel, P. 1956. Les Zygophyllacees de l, Afrique du Nord et du Sahara. Travaux de l'Institut de Recherches Sahariennes 14: 23-84.

Perveen, A. \& Qaiser, M. 2006. Pollen flora of Pakistan-XLIX. Zygophyllaceae. Pakistan Journal of Botany 38(2): 225-232.

Rohlf, F.J. 1972. An empirical comparison of three ordination techniques in numerical taxonomy. Systematic Zoology 21: 271-280.

Rohlf, F.J. 1993. NTSYS-PC. Numerical taxonomy and multivariate analysis system. Version 1.80. New York, Applied Biostatistics Inc.

Sands, M. 1989. Balanitaceae. In: Hedberg I, Edwards S. eds. Flora of Ethiopia, III: 433-436. Ethiopia, Addis Ababa University and Sweden, Uppsala University.

Scholz, H. 1964. Zygophyllaceae. In: Melchior H, ed. A. Engler's Syllabus der Pflanzenfamilien, 12th edition, Vol. 2: 251-252. Berlin, Bornträger.

Sheahan, M.C. \& Chase, M.W. 1996. A phylogenetic analysis of Zygophyllaceae based on morphological, anatomical and rbcL DNA sequence data. Botanical Journal of the Linnean Society 122: $279-300$.

Sheahan, M.C. \& Chase, M.W. 2000. Phylogenetic relationships within Zygophyllaceae based on DNA sequences of three plastid regions, with special emphasis on Zygophylloideae. Systematic Botany 25: 371-384.

Sheahan, M.C. \& Cutler, D.F. 1993. Contributions of vegetative anatomy to the systematics of Zygophyllaceae R. Br. Botanical Journal of the Linnean Society113: 227-262.

Shimakura, M. 1973. Polynomorphs of Japanese plants (in Japanese). Bulletin of the Osaka Museum of Natural History 5:180.

Soltis, D.E.; Soltis, P.S.; Chase, M.W.; Mort, M.E.; Albach, D.C.; Zanis, M.; Savolainen, V.; Hahn, W.H.; Hoot, S.B.; Fay, M.F.; Axtell, M.; Swensen, S.M.; Prince, L.M.; Kress, W.J.; Nixon, K.C. \& Farris, J.S. 2000. Angiosperm phylogeny inferred from $18 \mathrm{~S} \mathrm{rDNA}$, rbcL and atpB sequences. Botanical Journal of the Linnean Society133: 381-461.

Tackholm, V. 1974. Students' Flora of Egypt. Beirut, Cairo University.

Takhtajan, A. 1996. Diversity and classification of flowering plants. New York, Columbia University Press.

Takhtajan, A. 1969. Flowering plants: origin and dispersal. Edinburgh, Oliver and Boyd.

Takhtajan, A. 1980. Outline of the classification of the flowering plants (Magnoliophyta). Botanical Review 46: 225-359.

Takhtajan, A. 1983. The systematic arrangement of dicotyledonous families. Pp. 180-201. In: Metcalfe C.R. \& Chalk L. (Eds.). Anatomy of the Dicotyledons. 2nd ed. vol. 2: Oxford, Clarendon Press.

Takhtajan, A. 1986. Floristic regions of the world. Berkeley, University of California Press.

Thorne, R.F. 1992. An updated phylogenetic classification of the flowering plants. Aliso 13: 365-389.

Van den Berg, R.G. \& Groendijk-Wilders, N. 1999. Numerical analysis of the taxa of series Circaefolia (Solanum sect. Petota). Pp. 213-226. In: Nee, M., Symon, D., Lester, R., Jessop, J. (Eds.) Solanaceae IV. Kew, Royal Botanic Garden.

Van Huyssteen, D.C. 1937. Morphologisch-systematische Studien über die Gattung Zygophyllum. Berlin, Dissertation.

Van Zyl, L. 2000. A Systematic Revision of Zygophyllum in the Southern African Region. Ph.D. thesis, University of Stellenbosch.

Wieringa, J.J. 1990. Monopetalanthus exit. A systematic study of Aphanocalyx, Bikinia, Icuria, Michelsonia and Tetraberlinia (Leguminosae, Caesalpinioideae). Wageningen Agriculture University Papers 99(4): 1-320.

Yunus, D.\& Nair, P.K.K. 1988. Pollen morphology of Indian Geraniales. V. XV-XVI.1-22. New Dehli, Today and Tomorrow's Printer Publishers.

Zohary, M. 1972. Flora Palaestina 2. Jerusalem, Israel Academy of Sciences and Humanities, Pp. 247-252. 


\section{Appendix 1.}

List of specimens used for the study; species arranged alphabetically within subfamilies according to Engler (1896a, 1931) and Sheahan \& Chase $(1996,2000)$.

\section{Subfamily Balanitoideae}

Balanites aegyptiacus Delile

Egypt: Red sea coast, Wadi El Gemal, Abdel Khalik s.n. (SHG); Gebel Elba, W. Yahameib, Abdel Ghani \& Abdel Khalik s. n. (SHG); Red sea coast, Mersa Alam, gebel Hamata, Abdel Khalik et al. s. n. (SHG); Shalateen area, Wadi Baaneed, Abdel Khalik \& al. s. n. (SHG).

\section{Subfamily Peganoideae \\ Peganum harmala L.}

Egypt: Burg El Arab, Abdel Khalik s.n. (SHG); Ras El Hekma, Abdel Khalik, s.n. (SHG); Amria, Burg El Arab, Ibrahim El Sayed s.n. (CAI); Salloum town, Tackholm et al. s.n. (CAI); El Garawla, East Matrouh, Tackholm et al. s.n. (CAI); South Sinai, St. Catherine, H. Hosni s.n. (CAI); El Daba, A. Abdel Fadeel s.n. (CAI); Cairo- Suez road, Tackholm et al. s.n. (CAI); Wadi Gebal and Gebel Serbal region, Fayed et al. s.n. (SHG).

\section{Subfamily Seetzenioideae}

\section{Seetzenia lanata (Willd.) Bullock}

Egypt: South Sinai, St. Catherine, wadi El Arbaeen, Abdel Khalik s.n. (SHG).

Saudi Arabia: Mecca-Taif, El Hada road, Abdel Khalik \& Kotob Faghaly s.n. (SHG); Qassim, Near Nabhaniyah. $100 \mathrm{~km}$ SWof Buraydah. in hard gravelly sand. $2300 \mathrm{ft}$., Collenette1787

\section{Subfamily Tetradiclidoideae}

Tetradiclis tenella Litv.

Egypt: Alexandria, El Dekhala, Abdel Khalik \& El Kordy s.n. (SHG); Burg El Arab, Abdel Khalik \& El Kordy s.n. (SHG).

\section{Subfamily Tribuloideae}

Tribulus bimucronatus Viv.

Egypt: Gebel Elba, W. Yahameib, Abdel Ghani \& Abdel Khalik s.n. (SHG); Wadi abu Hammadah, Kom Ombo, tackholm et al. s.n. (CAI).

Saudi Arabia: Mecca, El Abdia, Umm Al Qura University campus, Abdel Khalik s.n. (SHG); Mecca - Jedda road, near Jedda, Abdel Khalik, s.n. (SHG); North Jedda, Abdel Khalik, s.n. (SHG).

\section{T. kaiseri Hosni}

Egypt: South Sinai, Farsh El Hamam, Kaiser 175 (CAIM); Sinai, Wadi El Siq, Kaiser 501 (CAI).

Saudi Arabia: Jedda, Abdel Khalik s.n. (SHG); MeccaEl Madina road, $70 \mathrm{~km}$ south El Madina, Abdel Khalik, s.n. (SHG).

\section{T. macropterus Boiss.}

Egypt: Cairo-Suez desert road, Abdel Khalik, s.n. (SHG); Cairo-Suez desert road, Tackholm et al. s.n. (CAI); Gebel Ahmar, Tackholm et al. s.n. (CAI); El Qossir, Drar 44 (CAIM).

Saudi Arabia: Mecca- Jedda road, $14 \mathrm{~km}$ from Jedda, Abdel Khalik, s.n. (SHG); Wadi Hanifa, Kadry \& Khodir, s.n. (CAI).

T. mollis Ehrenb. ex Schweinf.

Egypt: Wadi El Hamura, Shabetai 4207 (CAIM); Affluent of Wadi Adendan, Tackholm 259 (CAI); Abu Zaabal, Shabetai 6792 (CAIM); Cairo- Suez desert road, Oliver s.n. (CAI).

\section{T. pentandrus Forssk.}

Egypt: Cairo- suez road, Abdel Khalik, s.n. (SHG); Sohag University campus, near faculty of Science, Abdel Khalik, s.n. (SHG); Gift- El Qossir desert road, Abdel Khalik \& Osman s. n. (SHG); 18 km South of Mersa Alam, Tackholm et al. s.n. (CAI).

Saudi Arabia: Mecca, wadi Fatma, Abdel Khalik, s.n. (SHG); Jedda, Abdel Khalik, s.n. (SHG); Badr, Batanouni 132 (CAI).

\section{T. terrestris $\mathrm{L}$.}

Egypt: Gebel Elba, W. Yahameib, Abdel Ghani \& Abdel Khalik s.n. (SHG); Sohag, near the Nile, Abdel Khalik, s. n. (SHG); Wadi El Gemal, Abdel Khalik \& el kordy, s.n. (SHG); Burg El arab, Abdel Khalik, s.n. (SHG); South Sinai, Wadi Gebal, Abdel Khalik \& Osman s. n. (SHG); Gebel Serbal, Fayed et al. s.n. (SHG).

\section{Subfamily Zygophylloideae}

\section{Fagonia arabica $\mathrm{L}$.}

Egypt: Cairo-Alexandria desert road, Abdel Khalik s.n. (SHG); Cairo-Alexandria desert road, Tackholm et al. s.n. (CAI); Abu Rawash desert, El hadidi s.n. (CAI); Siwa Oasis, A. Zahran, s.n. (CAI); Cairo-Suez road, Kassas et al. s.n. (CAI); Wadi Natroun, Mustafa Imam s.n. (CAI); Faiyum, Chrtek s.n. (CAI); Helwan, Wadi Gerawi, Tackholm et al. s.n. (CAI); Gebel Serbal, Ain Alloza, Fayed et al. s.n. (SHG); Farsh Al Rommana, Fayed et al. s.n. (SHG).

\section{F. boveana (Hadidi) Hadidi \& El-Garf}

Egypt: Sinai, Wadi Ferieh, Tackholm et al. s.n. (CAI); Wadi Aber near Suez, Hadidi s.n. (CAI); Wadi Segal, N. Galala, M. Imam, s.n. (CAI); Wadi Abar, G. Ataqa, Suez, Imam \& Fadeel s.n. (CAI); Suez Gulf, Aboud,s Factory, Hadidi s.n. (CAI); Near Ain Soukhna, Suez Gulf, Wadi Segal, Hadidi s.n. (CAI); Wadi Sermatai, Abdel Khalik \& El Kordy s.n. (SHG); Sinai, Wadi Ferieh, Tackholm et al. s.n. (CAI); S. Sinai, Wadi El-Kid, Fayed et al. s.n. (SHG).

\section{F. bruguieri DC.}

Egypt: Wadi Hof, I. El Garf s.n. (CAI); cairo- Suez desert road, 102 from Cairo, Tackholm et s.n. (CAI); Suez, Wadi Batar, Tackholm et s.n. (CAI); north of suez, wadi fool, Tackholm et s.n. (CAI); Wadi Aber, Gebel Ataqa, Hadidi et al. s.n. (CAI); Wadi El Deir, S. Galala, 
Boulos s.n. (CAI); gebel Ahmar, Tackholm et s.n. (CAI); Helwan, Ibrahim El sayed s.n. (CAI); S. Sinai, Wadi Mander, Fayed et al. s.n. (SHG); Wadi Baida, Fayed et al. s.n. (SHG).

\section{F. cretica $\mathrm{L}$.}

Egypt: Alexandria-Matrouh coastal road, Sidi Abdel Rahman, Abdel Khalik s.n. (SHG); Sallum near shore, Tackholm et al. s.n. (CAI); Maruit, Mandara, Hefnawy s.n. (CAI); Maruit, Amal Amine s.n. (CAI); Ikingi Maruit, G. tackholm s.n. (CAI); $75 \mathrm{~km}$ from Alexandria, Cairo-Alexandria dearest road, Hadidi s.n. (CAI); Sidi AbdelRahman, Mexmuller et al. s.n. (CAI); ras El Hekma, Maruit, Boulos s.n. (CAI).

\section{F. glutinosa Delile}

Egypt: N. Sinai, Gebel El Maghara, Hadidi \& El Garf s.n. (CAI); N. Sinai, Rafah, El Garf s.n. (CAI); Sinai, St. Khaterina Mts. Gebel Serbal, Fayed et al. s.n. (SHG); Cairo-Suez road, K. 35, Imam s.n. (CAI); Cairo-Suez road, K. 22, Samir Ghabbour s.n. (CAI); Cairo-Suez road, K. 30, Imam s.n. (CAI); Cairo-Suez road, Kassas s.n. (CAI); Wadi Batar, Suez road, Tackholm et al. s.n. (CAI);Burg El Arab, Tackholm et al. s.n. (CAI).

\section{F. indica Burm.}

Egypt: Wadi Allaqi, S. the village, Abdallah s.n. (CAIM); Wadi Nugdeib, Tackholm et al s.n. (CAI); Kom Ombo desert, Wadi Khareit, Tackholm et al. s.n. (CAI); $60 \mathrm{~km}$ from Idfu, Idfu-Mersa Alam road, Hadidi \& El Garf s.n. (CAI); $90 \mathrm{~km}$ from Idfu, Idfu-Mersa Alam road, Tackholm et al. s.n. (CAI); Qena forest, Abbas \& Khattab s.n. (CAIM); Wadi Bir El Ain, Sohag, Abdel Khalik s.n.n (SHG); Kharga oasis, Boulaq, Imam s.n. (CAI); Kharga, Paris, at Omda's new spring, Tackholm \& Kassas 73 (CAI).

\section{F. isotricha Murb.}

Egypt: Gebel Elba, Wadi Mawaw, Abdel Khalik s.n. (SHG); Wadi Aak, Tackholm et al. 780 (CAI); Gebel Astriba, Tackholm et al. 956 (CAI); Wadi Halos, Red sea coast, Tackholm et al. 266 (CAI); Gebel Hamata, Tackholm et al. 329 (CAI).

\section{F. latifolia Delile}

Egypt: Cairo-Suez desert road, kmo 34, Samir Ghabbour s.n. (CAI); Wadi Anqabiya, El Hadidi s.n. (CAI); near the desert laboratory on the Suez road, Mustafa Imam s.n. (CAI); Suez road, K. 25 Tackholm s.n. (CAI); Suez road K. 35 Luckman Lawand s.n. (CAI); Cairo- Suez road, $50 \mathrm{~km}$ from Cairo, Abdel Khalik \& Abdel Ghani (SHG); Mountain near Wadi Er-Rokhama between Madi and Suez, G. Tackholm s.n. (CAI).

\section{F. mollis Delile}

Egypt: Galala desert, Wadi Hagaul, Hadidi \& Garf s.n. (CAI); Wadi Hof, G. Tackholm, s.n. (CAI); Wadi Rashid, Simpson 1069 (CAIM); Wadi Araba, Bir El Arayda, Shabetai 6589 (CAIM); Cairo - Suez road, Kassas s. N. (CAI); Wadi Aber, Gebel Ataqa, Tackholm et al. s.n. (CAI); N. Abu Zenima, S. Sinai, Abdallah 519 (CAIM); Wadi Gharandal, El Hadidi et al. s.n. (CAI).

\section{F. scabra Forssk.}

Egypt: Sallum Plateau, near the Town, Boulos s.n. (CAI); Burg El Arab, Hamada s.n. (CAI); Cairo- Alexandria desert road, Shabetai 7362 (CAIM); Abu Rawash, El Garf s.n. (CAI); Cairo-Suez road, $34 \mathrm{~km}$ from Cairo, El Hadidi s.n. (CAI); Wadi Liblab, near Cairo, Ayyad \& Amin s.n. (CAI); Gebel Ataqa, S. Suez, Hadidi s.n. (CAI); Gebel Ahmar, El Hadidi s.n. (CAI); Wadi Hamata, an affluent of Wadi Hoff, Galala desert, Garf s.n. (CAI); Wadi Hof, M. Hassib s.n. (CAI).

\section{F. sinaica Boiss.}

Egypt: North Sinai, Gebel Halal, Hadidi \& Garf s.n. (CAI); Wadi El Maghara, Boulos s.n. (CAI); Wadi El Arish, Drar s.n. (CAIM).

\section{F. tenuifolia Steud. \& Hochst.}

Egypt: Red Sea coast, Gebel Hamata, at the foot of the mountain, Tackholm et al. 444 (CAI).

\section{F. tristis Sickenb.}

Egypt: Between Mokattam and Gebel Ahmar, Hartmann s.n. (CAI); Gebel Tourrah, between Cairo and Helwan, Hartmann s.n. (CAI); Wadi Hof, Tackholm \& Hadidi, s.n. (CAI); Wadi Digla, Boulos s.n. (CAI); Wadi Aber, gebel Ataqa, S. Suez, Boulos s.n. (CAI); Wadi Rishrash, Kassas s.n. (CAI); Wadi sheikh Salma, near Wadi Gerawi, Tackholm et al. s.n. (CAI).

\section{Zygophyllum aegyptium Hosny}

Egypt: Alexandria- Matruh road, Burg El Arab, Abdel Khalik, s.n. (SHG); Mersa Matruh, Ghabbour s.n. (CAI); Alexandria, El Maks, Bornmüller 10504 (CAI); Burg El Brollus, Kassas s.n. (CAI); Baltim, Hadidi s.n. (CAI); Ras El Bar, Gazzar s.n. (CAI).

\section{Z. album L.}

Egypt: Mariut, Burg El Arab, Abdel Khalik \& Abdel Ghani s.n. (SHG); Red sea coast, mouth of wadi Gemal, Abdel Khalik \& El kordy s.n. (SHG); 80 km north of Mersa Alam, Tackholm et al. 574 (CAI); $73 \mathrm{Km}$ South of Mersa Alam, Tackholm et al. 28 (CAI); Mersa Samedai, Tackholm et al. 144 (CAI); Wadi Natroun, Chrtek (CAI); East Qantara, Gazzar s.n. (CAI).

Saudi Arabia: Red Sea coast, Jedda, Obher, Abdel Khalik s.n. (SHG); North of Jedda, on the coast, Abdel Khalik, s.n. (SHG).

\section{Z. coccineum $\mathrm{L}$.}

Egypt: Cairo-Alexandria desert road, $100 \mathrm{~km}$ from Cairo, Abdel Khalik s.n. (SHG); Wadi natroun, Abdel Khalik \& El Kordy s.n. (SHG); Sohag, wadi Bir El Ain, Abdel Khalik s.n. (SHG); Cairo - Suez desert road, $30 \mathrm{~km}$ from Suez, Abdel Khalik \& Abdel Ghani s.n. (SHG); Wadi El Arbaeen, South Sinai, Abdel Khalik \& Osman s.n. (SHG); Siwa Oasis, Boulos s.n. (CAI); Wadi Betar, Suez - Cairo road, Tackholm et al. s.n. (CAI); Wadi Hof, Tackholm et al. s.n. (CAI).

Saudi Arabia: Red Sea coast, Jedda, Obher, Abdel Khalik s.n. (SHG); North of Jedda, on the coast, Abdel Khalik, s.n. (SHG). 


\section{Z. decumbens Delile}

Egypt: Cairo- Suez desert road, $15 \mathrm{~km}$ from Cairo, Hilali et al. s.n. (CAIM); Cairo-Suez road, $93 \mathrm{~km}$ from Cairo, Darar s.n. (CAIM); Cairo-Suez road, Hadidi \& Hosny (CAI); Gebel Ataqa, Hadidi s.n. (CAI); Wadi Aber, Gebal Ataqa, Tackholm et al. s.n. (CAI); Wadi Qusseib, Kassas s.n. (CAI).

Z. dumosum Boiss.

Egypt: Wadi Heridin, south of El Arish, Darar s.n. (CAI); Darb El Hagg at Sudur el Heitam Darar 677 (CAIM).

\section{Z. simplex L.}

Egypt: Gebel Elba, W. Yahameib, Abdel Ghani \& Abdel Khalik s.n. (SHG); Siwa Oasis, Boulos s.n. (CAI); Wadi Natroun, Abdel Khalik s.n. (SHG); Cairo-Suez road, Tackholm et al. s.n. (CAI); Mersa Alam, Zahran et al. s.n. (CAI); Sohag, Wadi Bir El Ain, Abdel Khalik et al. s.n. (SHG).

Saudi Arabia: Jedda - El Madina road, Abdel Khalik s.n. (SHG); Wadi Noman, Mecca, Abdel Khaliks.n. (SHG); Mecca, El Abdia, Um-Alqura University campus, Abdel Khaliks.n. (SHG). 